\title{
Medical LED-on-the-Tip endoscope
}

D. Brüggemann, B. Blase, F. Bühs, R. Dreyer genannt Daweke, M. Kelp, H. Lehr, S. Oginski, S. Schlegel Department of Electromechanical and Optical Systems, Technical University Berlin, Berlin, Germany, brueggemann@fmt.tu-berlin.de

\section{Introduction}

Nowadays, external cold-light sources are illuminators for observing surgical sites using an endoscope during minimally invasive surgery. These light sources have several disadvantages as they are expensive, their lamps have a low durability as well as poor efficiency, and the fibre-optic cable to transport the light to the endoscope hinders handling of the unit.

\section{Methods}

Today’s High-Power-LEDs offer enough light to replace external cold-light sources. Within the endoscope, two locations for LEDs are conceivable. They can be placed in the handle where the generated heat is dissipated by its comparatively large surface. However, the light still has to be transported to the tip of the device, causing a loss of luminous flux. Alternatively, LEDs can be placed at the distal end of the endoscope where illumination is needed. In that case, the thermal energy has to be transported to the handle in order to achieve medical approval. This is effectively done by a heat transfer system consisting of heat pipes and copper connectors, so that the light of High-Power-LEDs with a "white spectrum” can be used without loss at the distal end of an endoscope.

\section{Results}

During research, comparison of different methods for transferring heat revealed that a set of heatpipes offers the optimal system to transport thermal energy within endoscopes. There is no need for additional elements like pumps to circulate a cooling fluid. Also, their very high thermal conductivity and their shape make heatpipes the ideal solution. Measurements on several laboratory prototypes at different alignments proved that they can keep temperatures within the limits specified in IEC 60601.

\section{Conclusion}

External cold-light sources for endoscope illumination have several disadvantages that can be overcome by an integrated light source consisting of LEDs. This calls for an adequate heat transfer systems which is successfully implemented by using heatpipes. 